\title{
Modeling propellant-based stimulation of a borehole with peridynamics
}

\author{
Rohan Panchadhara ${ }^{\mathrm{a}, *}$, Peter A. Gordon ${ }^{\mathrm{a}}$, Michael L. Parks ${ }^{\mathrm{b}}$ \\ ${ }^{a}$ ExxonMobil Research \& Engineering, 1545 Rt 22 E, Annandale, NJ-08801, USA \\ ${ }^{b}$ Center for Computing Research, Sandia National Laboratories, P.O. Box 5800, MS 1320, \\ Albuquerque, NM-87185, USA
}

\begin{abstract}
A non-local formulation of classical continuum mechanics theory known as peridynamics is used to study fracture initiation and growth from a wellbore penetrating the subsurface within the context of propellant-based stimulation. The principal objectives of this work are to analyze the influence of loading conditions on the resulting fracture pattern, to investigate the effect of in-situ stress anisotropy on fracture propagation, and to assess the suitability of peridynamics for modeling complex fracture formation.

It is shown that the loading rate significantly influences the number and extent of fractures initiated from a borehole. Results show that low loading rates produce fewer but longer fractures, whereas high loading rates produce numerous shorter fractures around the borehole. The numerical method is able to predict fracture growth patterns over a wide range of loading and stress conditions. Our results also show that fracture growth is attenuated with increasing in-situ confining stress, and, in the case of confining stress anisotropy, fracture extensions are largest in the direction perpendicular to the minimum compressive stress. Since the results are in broad qualitative agreement with experimental and numerical studies found in the literature, suggesting that peridynamics can be a powerful tool in the study of complex fracture network formation.
\end{abstract}

Keywords: peridynamics, dynamic fracture, borehole, in-situ stress, loading

\footnotetext{
${ }^{*}$ Corresponding author

Email address: rohan.panchadhara@exxonmobil.com (Rohan Panchadhara)
}

Preprint submitted to International Journal of Rock Mechanics 83 Mining Sciences December 22, 2016

(C) 2017. This manuscript version is made available under the Elsevier user license http://www.elsevier.com/open-access/userlicense/1.0/ 
rate

\section{Introduction}

Understanding fracture initiation and propagation in the subsurface is critical to the energy industry for improving productivity from reservoirs. In oil and gas reservoirs, production rates from wells may be enhanced through the process

5 of hydraulic fracturing, a well-stimulation technique [1. In hydraulic fracturing, fractures in the subsurface are created by pumping a mixture of fluid and sand into the borehole. Continuous pumping of the mixture increases the pressure inside the borehole up to the point when the fluid breaks into the formation and fractures the surrounding rock. As the mixture flows into the fractures, it induces continued growth and extension of the fractures away from the wellbore. Upon completion of the fracturing operation, the sand transported into the fractures keeps the fractures propped open to provide good flow conduits to the wellbore. A more detailed description may be found in [2]. It is generally accepted that the orientation and geometry of the induced hydraulic fractures

15 is controlled by the in-situ stress state, where initiation and growth occurs primarily in the direction perpendicular to the minimum confining stress in the rock. This results in a planar, bi-wing fracture geometry emanating from the wellbore. This type of stimulation greatly enhances the economics of producing so-called unconventional resources, including shale gas, tight gas and tight oil plays, where reservoir permeability is less than 1e-6 Darcy. As such resources represent a substantial portion of the industry's resource base, the continued development of well stimulation technology, as well as discovery and implementation of innovative and economical fracturing techniques is of increasing interest.

Propellant-based stimulation has also shown promise in enhancing connectivity between the wellbore and reservoir. In propellant-based stimulation, the ignition and controlled burning of a propellant generates a rapid increase in pressure in the wellbore due to the formation of gaseous combustion byprod- 
ucts. The rate of increase and magnitude of the peak pressure can far exceed that encountered in hydraulic fracture operations. The loading conditions for the propellant fracturing technique are characterized by peak pressures in the range of 10000 psi with a rise time on the scale of $\approx 1^{-2} \mathrm{~s}$, or a loading rate of $\approx 10^{6} \mathrm{psi} / \mathrm{s}$.

By contrast, hydraulic fracturing occurs at lower pressures, and the rate of loading is typically orders of magnitude slower. The principal effect of the high rate of loading in the propellant-based stimulation is that multiple fractures can initiate from the wellbore, in directions not necessarily aligned with the minimum in-situ confining stress [3, 4, 5, 6]. By comparison, the pressure profile corresponding to a typical explosive pulse is also shown, where loading rates

40 can reach $10^{12} \mathrm{psi} / \mathrm{s}$. Here, the peak pressures and loading rates are so high that it results in pulverization of the rock near the wellbore instead of fracture extension, not optimal for the goal of extending high conductivity pathways from the wellbore into the reservoir.

In propellant-based fracturing, it is generally accepted that two types of loading are in operation [7, 8, Early stage damage is driven by dynamic fracture processes. The detonation of the combustible material generates a stress wave that travels out from the borehole into the surrounding rock. This initially produces large compressive stresses that can exceed the rock strength, resulting in pulverization of the rock close to the borehole. As the stress wave moves

${ }_{50}$ further into the surrounding rock it induces a tensile hoop stress than can initiate and extend fractures radially out from the wellbore. The growth of some of these fractures may be attenuated by the competing growth process of nearby radial fractures. The selective extension of some subset of the initiated fractures is governed by a complex interaction of the local stress state near the fracture 55 tips, material heterogeneity and the in-situ stress state in the rock. 8

Following these dynamic processes, the fractures will be pressurized by the gas produced from the combustion process, which serves to further extend them from the wellbore. It is generally assumed that this process proceeds quasistatically as the loading rates from the gas moving along the fracture is slow 
relative to stress wave velocities in the rock. The relative importance of each of these mechanisms in determining the final fracture pattern clearly depends on the in-situ stress state, and the rate and nature of the loading process, as has been demonstrated by several theoretical and experimental studies. [3, 7, 8, 9, 10, 11 .

Given the complexity of the mechanisms that may control fracture initiation and growth, there has been considerable effort applied to numerical modeling of the overall process. Many of these efforts have been motivated by rock blasting for mining applications, and have focused on either discrete element methods (DEM [7]) or continuum-based approaches such as finite element methods (FEM [12]).

In the DEM approach, elements are bonded to their neighbors with an interaction law that contains a suitable rupture criterion to represent fracture between the elements. The method is specifically designed to model problems where discontinuities such as fractures may form. A few representative ap75 plications to which DEM models have been applied include the investigation of the interaction of stress waves on initiation and propagation of radial fractures [7, and wave propagation and fracture formation in rock under blasting conditions [13. More recently, Fakhimi et al 14] combined DEM with a Smooth Particle Hydrodynamics (SPH) model to examine the combined influence of the 80 elastic stress wave and gas pressurization on rock blasting.

Finite element approaches have also been directly applied to problems of dynamic fracture. Like DEM, the models will assume fracture occurs on element boundaries, and utilizes a suitable interaction and rupture criterion to describe the fracture process. Cho and Kaneko 12 permit elements to crack based on a non-linear tensile stress threshold and a Mohr-Coulomb compressive fracture criterion. Ma and An [15] utilized the Johnson-Holmquist model, a continuum damage model for brittle materials, within the commercial FEM package LSDYNA to simulate basting-induced rock fracture.

One of the difficulties that plague both DEM and FEM approaches is the need to prescribe the fracture plane of failure. When the failure planes are 
defined on element boundaries or at particle interfaces, the potential of mesh or particle size effects to influence simulation results becomes possible. Generalized or Extended Finite Element Methods [16] are capable of removing such mesh dependencies, but the techniques are limited to relatively simple fracture geometries, and usually cannot handle large scale initiation, branching and coalescence typified in dynamic fracture processes. Ideally, a numerical model for simulating dynamic fracture should be capable of accurately predicting aspects of fracture initiation, propagation, and bifurcation without an explicit reliance on the domain discretization to define the failure process.

A promising method that appears capable of overcoming some of these difficulties is peridynamics. Peridynamics [17] recasts classical continuum mechanics in a non-local framework, where points in a domain interact with its surrounding over a defined lengthscale. The conservation of momentum equation is reformulated so as to replace the traditional spatial derivatives with suitable integral counterparts. This greatly facilitates the description of discontinuities, and removes the requirement to explicity define fractures in terms of interfaces between a mesh or discrete particles.

The method has been shown to be particularly well suited for modeling dynamic fracture [18]. Many important features of dynamic fracture propagation have been validated against experiment with the technique, including crack path, propagation speeds, and bifurcation transitions [19, 20]. Early applications of the theory were restricted to simple brittle materials, but later extensions [21] enabled the development of a wide range of material models [22, 23].

The objective of this work is to investigate the effectiveness of peridynamics to model the influence of in-situ stress state and loading conditions on resultant fracture networks obtained from dynamic loading of a borehole. We restrict our attention to investigating the effect of the elastic stress waves on dynamic fracture patterns near the borehole. We begin with an overview of the peridynamic theory and methodology employed to model borehole fracture. We investigate 120 a wide range of loading and in-situ stress conditions, looking at the influence of loading rate, peak loading and confining stress anisotropy on resulting fracture 
patterns. Finally, we compare some of our results to similar experimental and numerical studies published in the literature.

\section{Model \& methods}

\subsection{Peridynamics model}

In the classical continuum solid mechanics, conservation of momentum for a body is given by

$$
\rho_{0}(\mathbf{x}) \ddot{\mathbf{u}}(\mathbf{x}, t)=\nabla \cdot \boldsymbol{\sigma}+\mathbf{b}(\mathbf{x}, t),
$$

where $\mathbf{x}$ is a point in the body, $\mathbf{u}(\mathbf{x}, t)$ is the displacement of point $\mathbf{x}$ at time $t$, $\rho_{0}$ is the mass density in the undeformed body, and $\mathbf{b}$ is an external body force. Here, $\nabla$ is the gradient operator taken with respect to the material coordinates and $\sigma$ is the Cauchy stress. As spatial derivatives are not defined on fracture surfaces and other discontinuities, Eq. (1) cannot be directly evaluated at these points. In peridynamics, Eq. (1) is reformulated so as to replace the traditional spatial derivatives with suitable integral counterparts, as the integral equations of the peridynamic theory can be applied to fracture solutions without any mathematical difficulty. We provide a brief introduction to the peridynamic theory here; see [21, 17, 24] for additional details.

The peridynamic equation of motion is

$$
\rho_{0}(\mathbf{x}) \ddot{\mathbf{u}}(\mathbf{x}, t)=\int_{\mathcal{H}_{\mathbf{x}}} \mathbf{f}\left(\mathbf{x}^{\prime}, \mathbf{x}, t\right) d V_{\mathbf{x}^{\prime}}+\mathbf{b}(\mathbf{x}, t) .
$$

Here, $\mathcal{H}_{\mathbf{x}}$ is a spherical region of radius $\delta$ centered at $\mathbf{x}$ and is the family of $\mathbf{x}$ and the vector valued function $\mathbf{f}$ is the force density per volume squared that $\mathbf{x}^{\prime}$ exerts on $\mathbf{x}$. Referring to Fig. 1(a) Eq. 22) supposes that point $\mathbf{x}$ interacts directly with all points that lie within a distance $\delta$ of $\mathbf{x}$. In this paper, we refer to $\delta$ as the peridynamic horizon.

The vector between $\mathbf{x}$ and any other point in its family is called a bond, defined as $\boldsymbol{\xi}=\mathbf{x}^{\prime}-\mathbf{x}$. Each bond has pairwise force density vector $\mathbf{f}\left(\mathbf{x}^{\prime}, \mathbf{x}, t\right)$ applied at both points. The force density is determined jointly by collective 


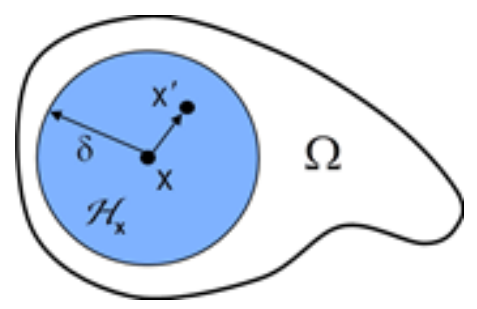

(a) Example peridynamic domain

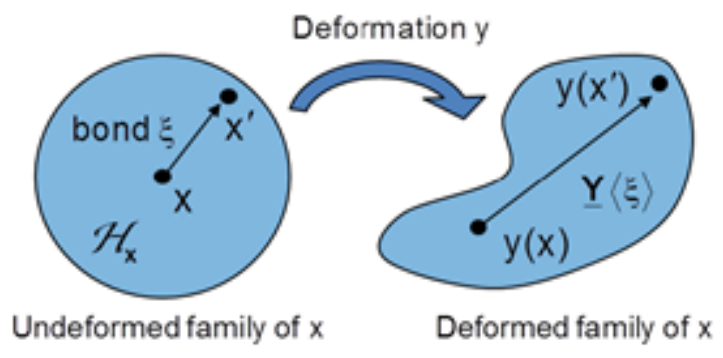

(b) Deformation state $\underline{\mathbf{Y}}\langle\boldsymbol{\xi}\rangle$ acting on bond $\boldsymbol{\xi}$

Figure 1: Subfigure (a) Each point $\mathbf{x}$ in the body $\Omega$ interacts directly with all points in the sphere $\mathcal{H}_{\mathbf{x}}$ of radius $\delta$ (the family of $\mathbf{x}$ ). Subfigure (b) The deformation state $\mathbf{Y}$ maps a bond $\boldsymbol{\xi}$ into its deformed configuration at time $t$.

deformation of $\mathcal{H}_{\mathbf{x}}$ and collective deformation of $\mathcal{H}_{\mathbf{x}^{\prime}}$. Bond forces are antisymmetric, meaning that $\mathbf{f}\left(\mathbf{x}^{\prime}, \mathbf{x}, t\right)=-\mathbf{f}\left(\mathbf{x}, \mathbf{x}^{\prime}, t\right)$.

Peridynamic material models are frequently written in terms of mathematical objects called states, which we briefly describe here. For the purposes of our discussion, states are operators that act on the family of a point $\mathbf{x}$. A vector state is an operator whose image is a vector, and may be viewed as a generalization of a second-rank tensor. Similarly, a scalar state is an operator whose image is a scalar. For a more complete discussion of states, see [21]. Referring to Fig. 1(b), we define the deformation state $\underline{\mathbf{Y}}\left\langle\mathbf{x}^{\prime}-\mathbf{x}\right\rangle=\mathbf{y}\left(\mathbf{x}^{\prime}\right)-\mathbf{y}(\mathbf{x})$, where $\mathbf{y}(\mathbf{x})$ denotes the position of point $\mathbf{x}$ at time $t$.

We note that $\mathbf{f}$ in 2 has contributions from material models at both $\mathbf{x}$ and $\mathbf{x}^{\prime}$. We express this as

$$
\mathbf{f}\left(\mathbf{x}^{\prime}, \mathbf{x}, t\right)=\underline{\mathbf{T}}[\mathbf{x}, t]\left\langle\mathbf{x}^{\prime}-\mathbf{x}\right\rangle-\underline{\mathbf{T}}\left[\mathbf{x}^{\prime}, t\right]\left\langle\mathbf{x}-\mathbf{x}^{\prime}\right\rangle,
$$


densities. $\underline{\mathbf{T}}$ is determined by the constitutive model $\underline{\mathbf{T}}=\widehat{\mathbf{T}}(\underline{\mathbf{Y}})$, where $\widehat{\mathbf{T}}$ maps the deformation state to the force state.

In this work, we use a simple interaction model known as the prototype micro-elastic brittle (PMB) [18 material model. In this representation, the force between two points $\mathbf{x}^{\prime}$ and $\mathbf{x}$ is proportional to the bond stretch $\mathrm{s}$,

$$
s=\frac{\|\underline{\mathbf{Y}}\langle\boldsymbol{\xi}\rangle\|-\|\boldsymbol{\xi}\|}{\|\boldsymbol{\xi}\|}
$$

Bond stretch is a dimensionless quantity that acts as a one-dimensional metric of strain. It measures the relative elongation of the deformed bond relative to the undeformed state $\boldsymbol{\xi}$. At equilibrium, the bond stretch $s=0$.

The PMB model is expressed using the force vector state field

$$
\underline{T}[\mathbf{x}]\langle\boldsymbol{\xi}\rangle=\left\{\begin{array}{cc}
\frac{1}{2} c s \frac{\mathbf{Y}}{\| \underline{\xi}\rangle}\langle\boldsymbol{\xi}\rangle \| & s<s_{0} \\
0 & s \geq s_{0}
\end{array},\right.
$$

As depicted in Fig. 2(a), the force acts along the direction of the deformed bond vector and is proportional to the micromodulus, $c$, which represents the bond stiffness. Bonds can sustain a maximum tensile elongation, denoted by the critical bond stretch $s_{0}$, before they rupture irreversibly. Once the bond between $\mathbf{x}$ and $\mathbf{x}^{\prime}$ bond ruptures, the bond is considered damaged and loses the ability to transmit force between the points. Forces acting at $\mathbf{x}$ (and $\mathbf{x}^{\prime}$ ) redistribute to other bonds within their horizons that have not yet broken.

In a typical simulation, the loading of the peridynamic body will induce a progression of damaged bonds. The total bond breakage can be represented at a given point by a scalar damage quantity, defined as the ratio of the number of broken bonds in the current configuration to the number of bonds in the original undeformed state. Damage at a point can vary from 0 (completely intact) to 1 (all bonds broken), and an example is shown in Fig. 2(b). While damage is inherently a non-local quantity, note that there is a natural correspondence 175 between the maximum in the damage field and the identification of the fracture path. 


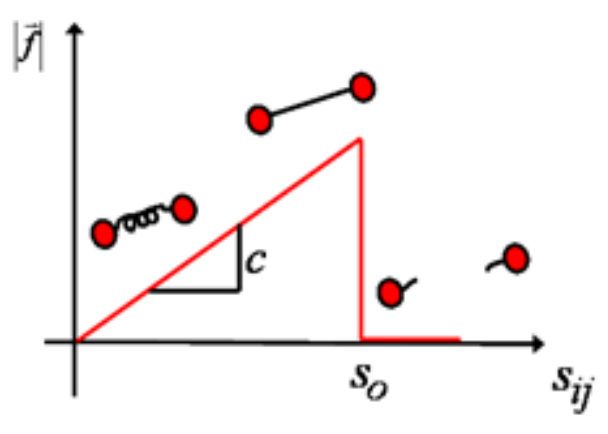

(a)

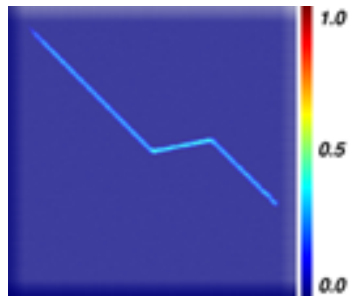

(b)

Figure 2: Subfigure (a) Interaction law for the PMB material. Subfigure (b) Representation of fracture through scalar damage field in a peridynamic body.

A concrete description of the PMB material requires specification of the micromodulus and the critical bond stretch, both of which can be linked to physical properties of real materials. The micromodulus can be linked to the bulk modulus, $K$ by equating the elastic energy stored in a peridynamic body undergoing isotropic tension with that of the continuum linear elastic material counterpart. This equivalence yields

$$
c=\frac{18 K}{\pi \delta^{4}} .
$$

Similarly, the critical bond stretch can be linked to a material's resistance to fracture by equating the energy release rate, $G_{0}$ to the energy released in breaking all peridynamic bonds across a given cross-section of surface to be created,

$$
s_{0}=\sqrt{\frac{5 G_{0}}{9 K \delta}} .
$$

A more detailed derivation of Eqs. 6 and 7 can be found in [18.

Note that both $c$ and $s_{0}$ are expressed in terms of the horizon, $\delta$. Thus, one can produce PMB material models with differing degrees of non-locality that correspond to the same bulk physical properties. In this work, the horizon is set to a predetermined value and the lattice spacing $a_{0}$ is chosen to be $\delta / 3$. Several authors [20, 26] have performed convergence studies to demonstrate the influence of the ratio of the horizon to the lattice spacing on the resulting 
fracture patterns. Such a study is beyond the scope of this paper. The reader is referred to these articles for a detailed analysis.

\subsection{Borehole geometry and loading}

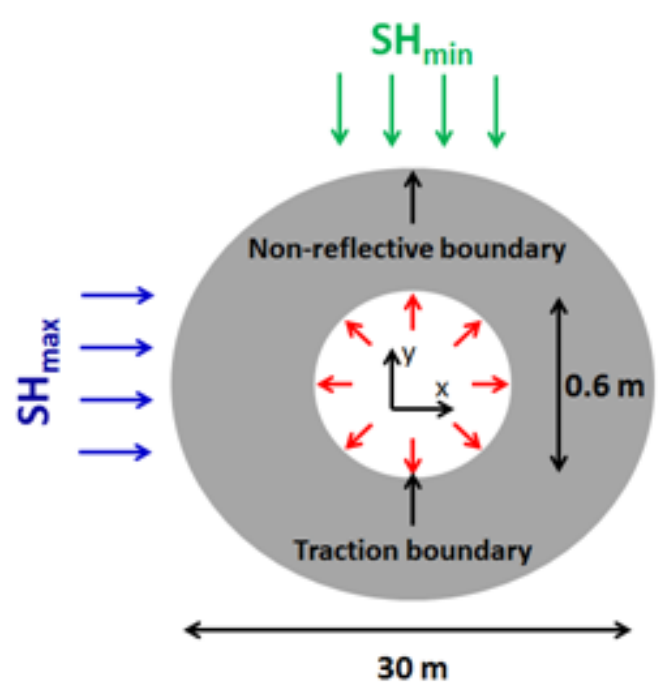

(a)

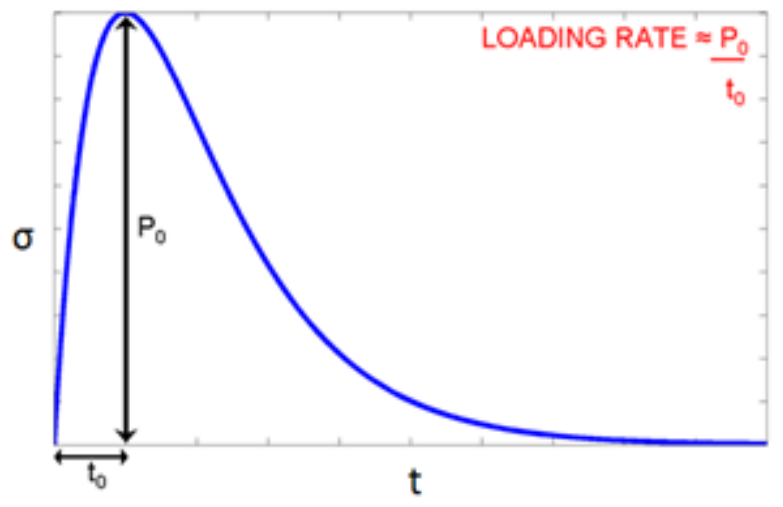

(b)

Figure 3: Subfigure (a) Schematic of the borehole geometry used in this study. Also shown are the boundary conditions imposed on the system. Not to scale. Subfigure (b) Schematic of the pressure waveform used to load the internal surface of the borehole.

Shown in Fig. 3(a) is a schematic of the borehole geometry used in this study. This simplified geometry consists of a cylindrical rock material of diameter $D$ $=10 \mathrm{~m}$ with a through borehole of diameter $d_{b}=0.6 \mathrm{~m}$. Relevant material properties of the rock are shown in Table 1. Heterogeneity is introduced in the rock model by randomly varying the fracture toughness in the domain by $\pm 10 \%$.

Pressurization of the borehole is introduced by the equation [12]

$$
\boldsymbol{P}_{\boldsymbol{b}}(t)=P_{0} * \frac{(\exp (-\alpha t)-\exp (-\beta t))}{\left(\exp \left(-\alpha t_{0}\right)-\exp \left(-\beta t_{0}\right)\right)}
$$

and is depicted schematically in Fig. 3(b) The load increases exponentially from zero at $t=0$ to a maximum load $P_{0}$ over a rise time $t_{0}$, then exhibits 


\begin{tabular}{cc}
\hline Property & Value \\
\hline Density $(\rho)$ & $2700.0 \mathrm{Kg} / \mathrm{m}^{3}$ \\
Bulk Modulus $(k)$ & $37.0 \mathrm{GPa}$ \\
Poisson's Ratio $(\nu)$ & 0.25 \\
Energy Release Rate $\left(G_{0}\right)$ & $298 \mathrm{~J} / \mathrm{m}^{2}$ \\
Rock Diameter $(D)$ & $30 \mathrm{~m}$ \\
Borehole Diameter $\left(d_{b}\right)$ & $0.6 \mathrm{~m}$ \\
\hline
\end{tabular}

Table 1: Material and geometric parameters used in the study.

a slower decline governed by the decay ratio $\beta / \alpha$. The loading rate for such a profile can be approximated as $\dot{L}=P_{0} / t_{0}$. This waveform provides ample flexibility to investigate the effect of loading rate, decay rate, and total energy input on the resulting fracture patterns. Simulations are performed using five loading conditions as shown in Table2. The first three are intended to illustrate the effect of the loading rate, whereas the last two cases illustrate the effect of the decay rate.

\begin{tabular}{ccccc}
\hline Case & $P_{0}(\mathrm{MPa})$ & $t_{0}(\mathrm{~ms})$ & $\beta / \alpha(\mathrm{ms})$ & $\dot{L}(\mathrm{MPa} / \mathrm{ms})$ \\
\hline 1 & 100.0 & 10.0 & 1.5 & 10.0 \\
2 & 100.0 & 1.0 & 1.5 & 100.0 \\
3 & 100.0 & 0.1 & 1.5 & 1000.0 \\
\hline 4 & 100.0 & 1.0 & 131.3 & 100.0 \\
5 & 100.0 & 0.1 & 2120.0 & 1000.0 \\
\hline
\end{tabular}

Table 2: Loading conditions applied on the borehole.

The rock is also assumed to be under a state of biaxial compression as shown in Fig. $3(\mathrm{a})$, with maximum and minimum compressive stresses denoted as $S H_{\max }$ and $S H_{\min }$, respectively. Under plane strain conditions, the equilibrium stress $\left(\boldsymbol{\sigma}_{r r}, \boldsymbol{\sigma}_{\theta \theta}, \boldsymbol{\sigma}_{r \theta}\right)$ and displacement $\left(\mathbf{u}_{r r}, \mathbf{u}_{\theta \theta}\right)$ fields for a linear elastic 
material near the borehole are given by [27]

$$
\begin{aligned}
\boldsymbol{\sigma}_{r r}(r, \theta)= & \boldsymbol{\sigma}_{a}\left(1-\left(\frac{r_{b}}{r}\right)^{2}\right)+\boldsymbol{P}_{\boldsymbol{b}}\left(\frac{r_{b}}{r}\right)^{2}+\boldsymbol{\sigma}_{d}\left(1-4\left(\frac{r_{b}}{r}\right)^{2}+3\left(\frac{r_{b}}{r}\right)^{4}\right)\left(1-2 \sin ^{2} \theta\right), \\
\boldsymbol{\sigma}_{\theta \theta}(r, \theta)= & \boldsymbol{\sigma}_{a}\left(1+\left(\frac{r_{b}}{r}\right)^{2}\right)-\boldsymbol{P}_{\boldsymbol{b}}\left(\frac{r_{b}}{r}\right)^{2}-\boldsymbol{\sigma}_{d}\left(1+3\left(\frac{r_{b}}{r}\right)^{4}\right)\left(1-2 \sin ^{2} \theta\right) \\
\boldsymbol{\sigma}_{r \theta}(r, \theta)= & \boldsymbol{\sigma}_{d}\left(1+2\left(\frac{r_{b}}{r}\right)^{2}-3\left(\frac{r_{b}}{r}\right)^{4}\right)(2 \sin \theta \cos \theta) \\
\mathbf{u}_{r r}(r, \theta)= & -\boldsymbol{P}_{\boldsymbol{b}} \frac{r_{b}^{2}}{2 G r}+\left(\frac{r_{b}}{4 G}\right)\left(\boldsymbol{\sigma}_{a}\left((\kappa-1)\left(\frac{r}{r_{b}}\right)+2\left(\frac{r_{b}}{r}\right)\right)\right. \\
& \left.+2 \boldsymbol{\sigma}_{d}\left(\left(\frac{r}{r_{b}}\right)+(\kappa+1)\left(\frac{r_{b}}{r}\right)-\left(\frac{r_{b}}{r}\right)^{3}\right) \cos 2 \theta\right) \\
\mathbf{u}_{\theta \theta}(r, \theta)= & -\left(\frac{r_{b}}{2 G}\right) \boldsymbol{\sigma}_{\boldsymbol{d}}\left(\left(\frac{r}{r_{b}}\right)+(\kappa-1)\left(\frac{r_{b}}{r}\right)+\left(\frac{r_{b}}{r}\right)^{3}\right) \sin 2 \theta
\end{aligned}
$$

where $\boldsymbol{\sigma}_{a}=0.5 *\left(S H_{\max }+S H_{\min }\right), \boldsymbol{\sigma}_{d}=0.5 *\left(S H_{\max }-S H_{\min }\right), r_{b}$ is the borehole radius, $G$ is the shear modulus, and $\kappa=3-4 \nu$, where $\nu$ is the Poisson's ratio. The outer boundary of the domain is constrained to the displacement fields of Eq.9, assuming an initial borehole pressure of zero, to model the effect of in-situ compression.

To examine the influence of in-situ stress state on fracture pattern, simulations are performed on four combinations of maximum and minimum compressive stress states as shown in Table 3 . The equilibrium stresses near the borehole under the in-situ stresses for case $\mathrm{C}$ is shown in Fig. 4 The stress fields are computed by quasi-static relaxation of the peridynamic body, and are compared with the analytical solution given by Eq. 9. The agreement in general is quite good, although we note some discrepancies in the radial and hoop stresses very close to the wellbore. This is due to the fact that the surface particles exhibit a somewhat lower effective stiffness than particles in the interior of the peridynamic body. No attempt to modify the surface particles was made in this work.

For the remainder of this report notation $[(\mathrm{A}, \mathrm{B}, \mathrm{C}, \mathrm{D})-(1,2,3,4,5)]$ is used to denote the corresponding case.

\subsection{Computational details}

220

Calculations were performed using the Peridynamic extensions 28] implemented in LAMMPS [29], and modifications therein. The domain was dis- 


\begin{tabular}{ccc}
\hline Case & $S H_{\max }(\mathrm{MPa})$ & $S H_{\min }(\mathrm{MPa})$ \\
\hline $\mathrm{A}$ & 0.0 & 0.0 \\
$\mathrm{~B}$ & 10.0 & 10.0 \\
$\mathrm{C}$ & 12.5 & 7.5 \\
$\mathrm{D}$ & 15.0 & 5.0 \\
\hline
\end{tabular}

Table 3: In-situ stress states imposed on the borehole.

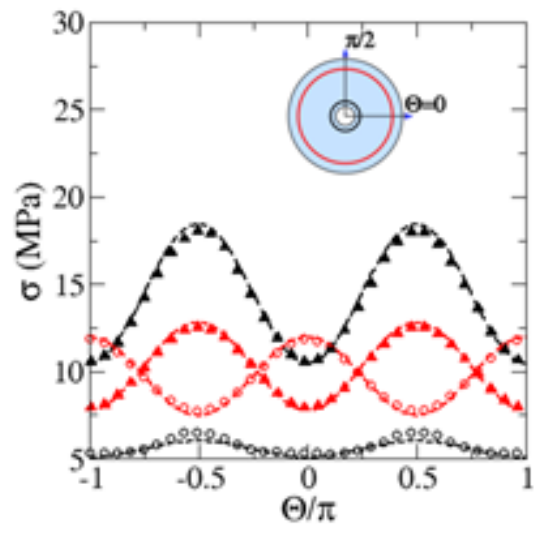

(a)

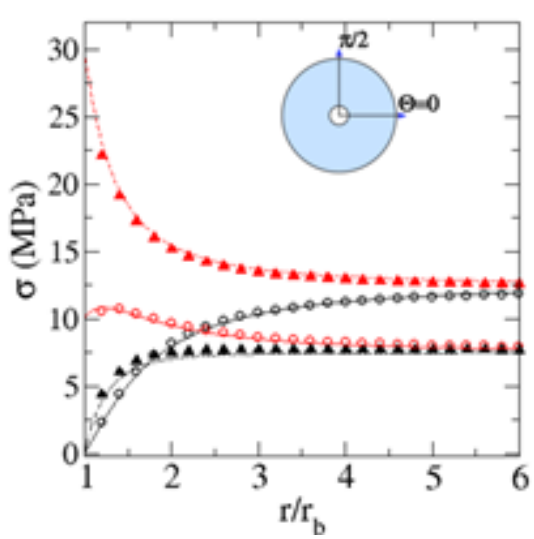

(b)

Figure 4: Stress fields obtained from quasi-static relaxation of peridynamics model for SHmax $=12.5 \mathrm{MPa}$ and SHmin = 7.5 MPa. Subfigure (a) $\bigcirc:\left.\sigma_{r r}\right|_{\frac{r}{r_{b}}}=1.5, \mathbf{\Delta}:\left.\sigma_{\theta \theta}\right|_{\frac{r}{r_{b}}}=1.5, \bigcirc:$ $\left.\sigma_{r r}\right|_{\frac{r}{r_{b}}=6}, \mathbf{\Delta}:\left.\sigma_{\theta \theta}\right|_{\frac{r}{r_{b}}=6}$. Subfigure (b) $\bigcirc:\left.\sigma_{r r}\right|_{\theta=0}, \mathbf{\Delta}:\left.\sigma_{r r}\right|_{\theta=\frac{\pi}{2}}, \bigcirc:\left.\sigma_{\theta \theta}\right|_{\theta=0}, \boldsymbol{\Delta}:\left.\sigma_{\theta \theta}\right|_{\theta=\frac{\pi}{2}}$. Lines represent the corresponding stresses given by the Kirsch solution computed from Eq. 9

cretized on a simple cubic lattice with edge length of $a_{0}=0.03 \mathrm{~m}$ with the origin at the center of the borehole as shown if Fig. 3(a). With the horizon equal to $3 a_{0}$, each particle in the bulk has 122 bonds. The discretized equations of motion are integrated using a velocity Verlet algorithm with a timestep of $1 \times 10^{-7} \mathrm{~s}$. Except where noted, the systems were evolved in time until no further damage evolution was observed.

In order to impose a given in-situ stress state on the peridynamic system, the system of equations given by Eq. 9 was solved for the displacement field.

This displacement was imposed on the system incrementally and the system 
was allowed to relax between increments to minimize the potential energy. The pressure pulse was imposed as additional forces acting on the innermost layer of peridynamic particles. Bonds between these particles were not allowed to break in order to ensure proper force transmission from the borehole to the rock. To mitigate the effects of wave reflections off the fixed outer boundaries, a viscous damping term was added to the equations of motion on a 1 meter cylindrical band of particles near the outer boundary. Minimal effects of reflections from the boundary were observed for all the simulations.

\section{Results and discussion}

240

\subsection{General features}

We first present some general features of the material response to rapid borehole loading. This is illustrated with case $[(\mathrm{A})-(1)]$. The initial application of load induces a compressive stress wave that increases in magnitude and propagates radially outward. The hoop stress also increases and is uniformly tensile, a consequence of the Poisson effect. Fig. 5 depicts contours of hoop stress and radial stress in a $6 \mathrm{~m}$ by $6 \mathrm{~m}$ portion of the domain centered at the borehole at three values of loading time, just before and after fracture initiation. Here, the hoop and radial stress components are calculated by post-processing the virial stress tensor [30]. At $t=0.3 \mathrm{~ms}$, prior to fracture initiation, the radial and hoop stress field is axisymmetric.

Upon further loading, bonds connected to the weakest peridynamic particles on the borehole surface eventually rupture, which breaks the symmetry of the stress field. This can be seen in the stress fields at $t=0.6 \mathrm{~ms}$ and beyond, where a highly correlated process of bond breakage emerges, which can be identified almost simultaneously at 4 locations and begin to propagate radially outward. The concentration in the hoop stress contours clearly identifies the location of the propagating fractures, as evident in Fig. 5. As the fractures propagate the material on either side of the fractures is compressed. This compression zone 
Hoop Stress
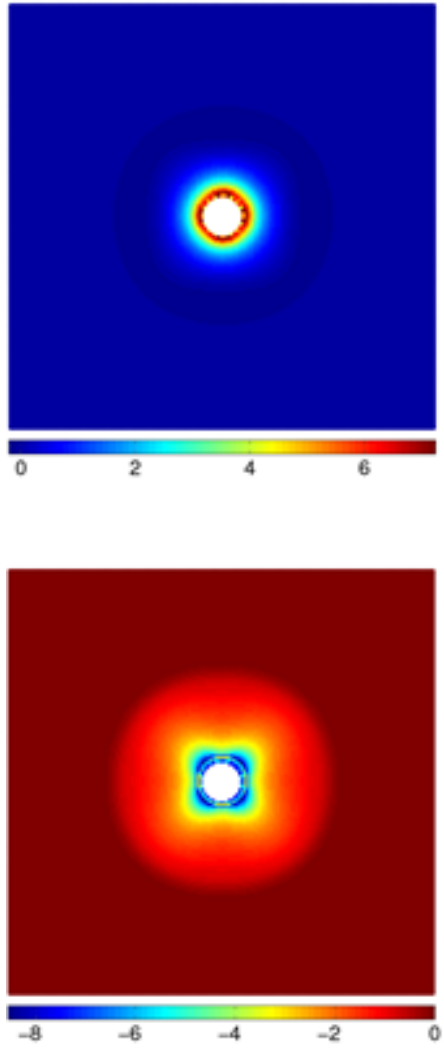

$\mathrm{t}=0.3 \mathrm{~ms}$

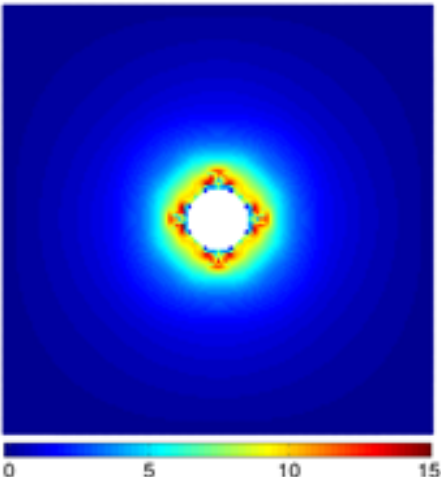

Radial Stress

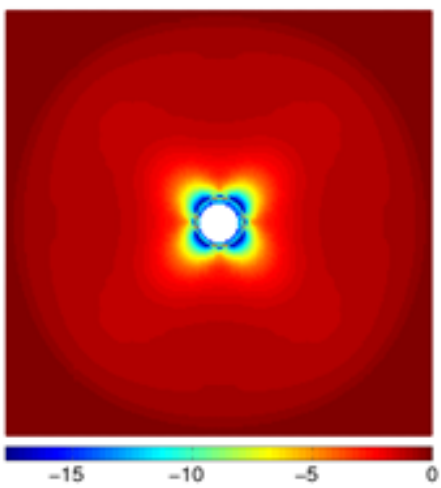

$\mathrm{t}=0.6 \mathrm{~ms}$
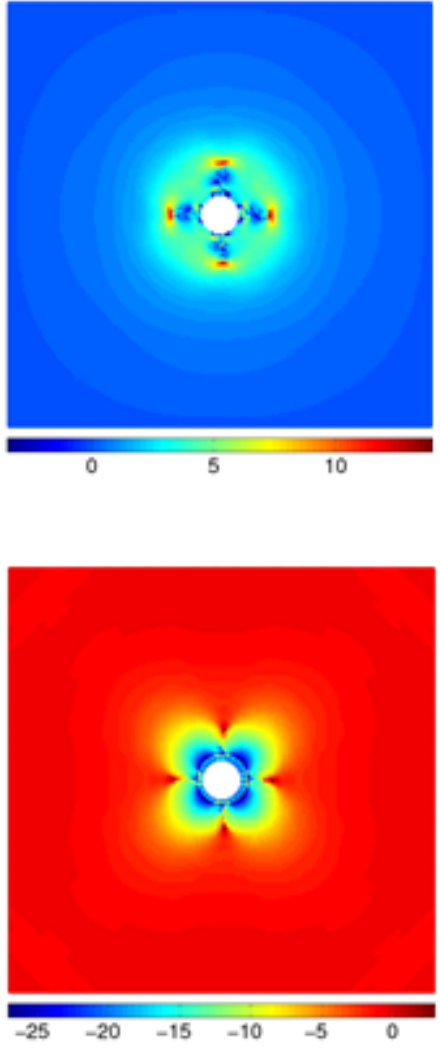

$\mathrm{t}=0.9 \mathrm{~ms}$

Figure 5: Contour of hoop stress and radial stress in a $6 \mathrm{~m}$ by $6 \mathrm{~m}$ portion of the domain centered at the borehole prior to and post fracture initiation for case [(A)-(1)]. Color bar is in $\mathrm{MPa}$

tends to inhibit initiation of additional fractures from the borehole surface. As the loading process continues, energy is supplied to the propagating crack tip from the continued loading of the borehole surface. The fractures arrest at $\approx$ $8 \mathrm{~ms}$, when the hoop stress near the fracture tips decreases and the pressure wave no longer delivers sufficient energy to continue the damage process.

The final state of the fractures is depicted through the damage contours in Fig. 6(a). By comparison, the same system was loaded to a peak pressure of 
100MPa through a series of small pressure increments, each increment followed by a relaxation of the potential energy. The final state of this quasi-static loading process is shown in Fig. 6(b) It is clear that the rapid loading imparts a strong inertial component to the fracture generation, resulting in much longer fractures. In addition, it is notable that the more strongly driven system results in straight fractures, whereas the fractures generated through quasi-static loading are more influenced by the local material heterogeneity, resulting in a more curved fracture path.

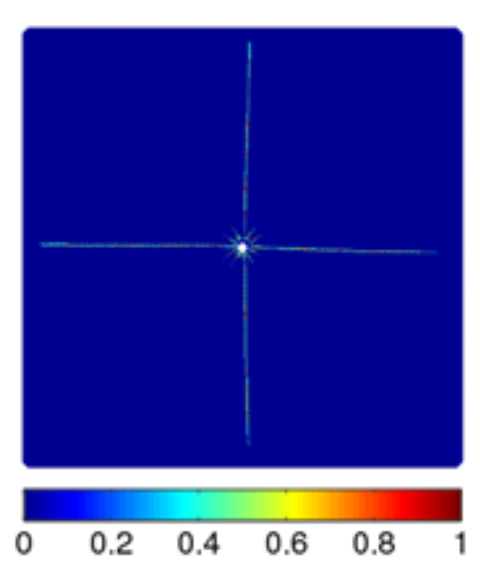

(a)

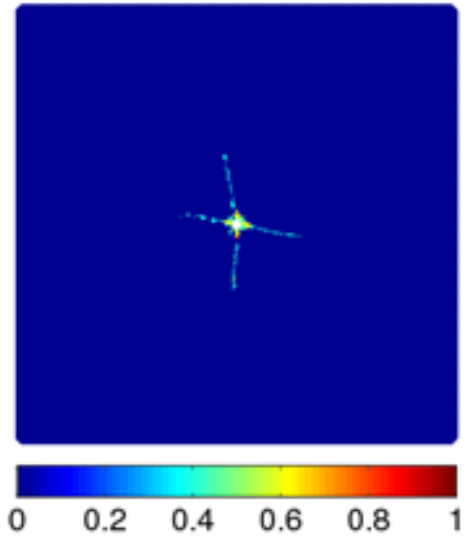

(b)

Figure 6: Damage contours in $10 \mathrm{~m}$ by $10 \mathrm{~m}$ domain around borehole at $100 \mathrm{MPa}$ loading with no confining stresses. Subfigure (a) case [(A)-(1)] at $t=10 \mathrm{~ms}$, Subfigure (b) same system quasi-statically loaded to $100 \mathrm{MPa}$.

\subsection{Influence of loading rate}

Shown in Fig. 7(a) are contours of damage for the initially unloaded cases [(A)-(1,2,3)]. The contours shown are at the time of maximum fracture extension. Also shown in Fig. 7(b) is the damage field in a $6 \mathrm{~m}$ by $6 \mathrm{~m}$ portion of the domain centered at the borehole. As is evident from the figures, marked differences exist in the resulting fracture patterns due to the variation in loading rate. First, higher loading rate produces more radial fractures while slow loading produces fewer and longer radial fractures. In addition, the fracture patterns 
for cases $[(\mathrm{A})-(2,3)]$ display extensive fracture branching, which is absent in the slowest loading case. Very close to the borehole surface, it is also apparent that increased loading results in a uniform ring of high damage. The size of this zone of extreme damage appears to grow with loading rate. This zone appears to be consistent with the formation of a crushed or pulverized zone typified under explosive loading conditions.

Strong differences can be identified in the early time behavior of each system just prior to fracture initiation. We focus on a behavior of the hoop stress near the borehole, as this should be a dominant indicator of nucleating tensile radial cracks. Fig. 8 depicts the temporal evolution of the hoop stress, averaged at a distance of $\mathrm{r}=2 r_{b}$ from the borehole. For the lowest loading rate considered, case $[(A)-(1)]$, the stress decreases after a short induction time, which reflects the time for the stress wave to propagate out from the wellbore. The hoop stress becomes tensile (negative) and continues to decrease until fracture initiation occurs, at which point the stress is released and rises up to nearly zero. Fig. 9(a) shows the hoop stress in the same ring, now as a function of orientation around the wellbore, at a series of time slices just prior to and after initiation. It can be seen that this distribution is essentially uniform prior to initiation (at $t=0.5$ and $0.7 \mathrm{~ms}$ ), but very quickly bifurcates into deep minima at $\theta=0,90,180$, and $270^{\circ}$. This coincides with the formation of the 4 fractures observed in Fig. 7(a).

The same examination for loading case (A)-(2) reveals qualitatively different behavior. First, the early time behavior of the averaged hoop stress shows that for a small period of time, the stress is compressive (positive), before rapidly changing direction and turning tensile. The rate at which the tensile stresses grow is much larger than case (A)-(1). Prior to initiation, (Fig. 9(b)), an oscillatory pattern in the hoop stress emerges, with minima at $\pm \frac{\pi}{4}$ and $\pm \frac{3 \pi}{4}$. Initiation events occur rapidly at $\approx t=0.25 \mathrm{~ms}$. It is worth noting that the hoop stress continues to remain tensile for a period of time as these nucleation events proceed, before the average hoop stress increases back toward zero. Case (A)-(3) exhibits many of the same features as case (A)-(2), although many of the trends are more pronounced. One notable difference is that the nucleation events that 
occur quickly release stress, and the average hoop stress more quickly reverse direction as a result of the nucleation events. It is also worth noting that in Fig. 8, regardless of loading rate, the time taken for the tensile hoop stress to dissipate from its maximum value to $\approx$ zero appears independent of loading rate; it takes about $0.3 \mathrm{~ms}$ for the stress relaxation process to occur.

These observations can help explain the fracture patterns observed in Fig. 7 There are two competing processes in operation that mediate the number of fractures generated around the borehole - the uniform tensile hoop stress from the borehole loading, and the localized stress redistribution that occurs upon fracture initiation. The initial nucleation of fractures occurs in response to the uniform tensile hoop stresses imparted from the borehole loading, and the 325 subsequent stress redistribution tends to compress both sides of the material around the plane at which fracture initiation occurs. When the loading rate is low, such as in case [(A)-(1)], the process of local stress redistribution is able to attenuate the additional tensile hoop stress created from the loaded borehole, and subsequent fracture nucleation is inhibited. At higher loading rates, however, the rate of increase, or inertia of the rising hoop stress from the borehole load cannot be easily attenuated by the stress shedding effects of a few nucleation events, and multiple fractures are able to form. This is observed in case [(A)-(2)] and [(A)-(3)] in Fig. 7, where fractures initiate from most of these damaged points and the high rate of borehole loading provides enough energy to propagate all of them.

A few other observations are evident. For all three cases, the fractures arrest after a certain time into the loading cycle. For case [(A)-(1)] the fractures arrest at time $t=t_{0}$, that is, at peak pressure in the loading cycle. For the other two cases the fractures arrest towards the end of the loading cycle. The ultimate fracture extensions depend significantly on how early during the initial loading phase preferential fracture initiation occurs. This may be because propagating fractures produce a zone of compression on either side of them which limits further fracture initiation in their vicinity, which in turn promotes more efficient transmission of energy to the propagating fracture tips. 

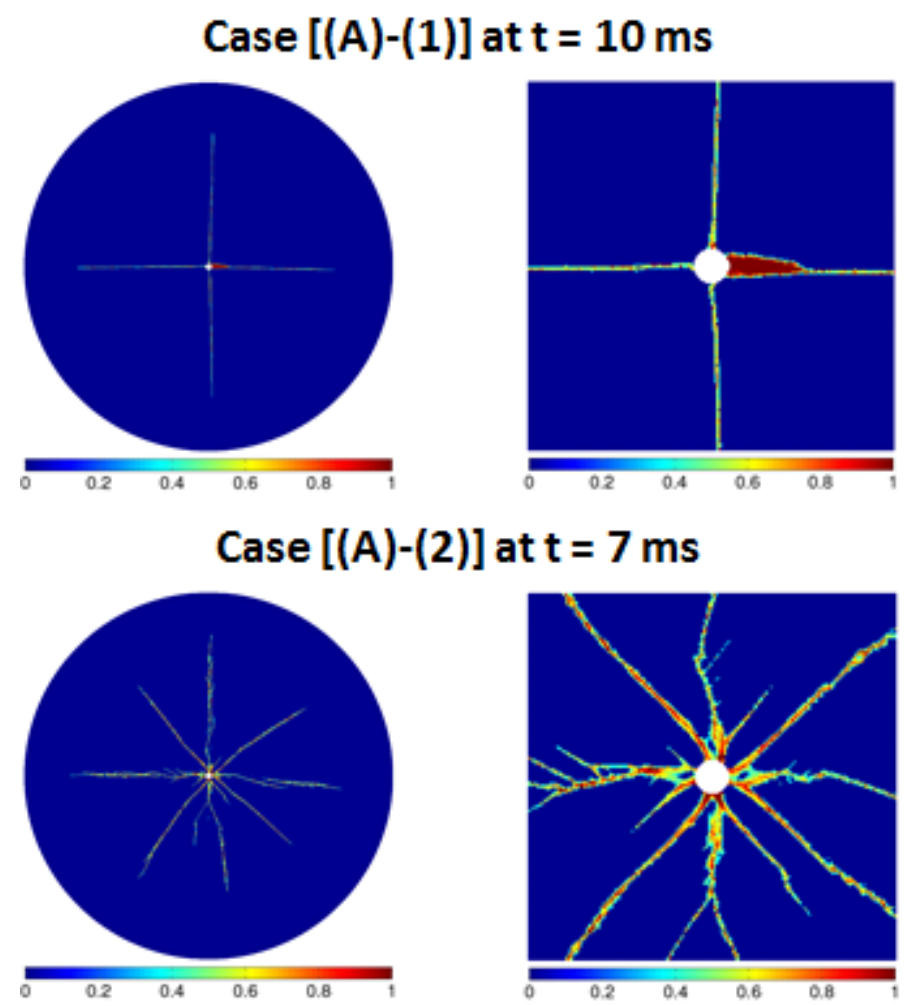

\section{Case $[(\mathrm{A})-(3)]$ at $\mathrm{t}=1 \mathrm{~ms}$}

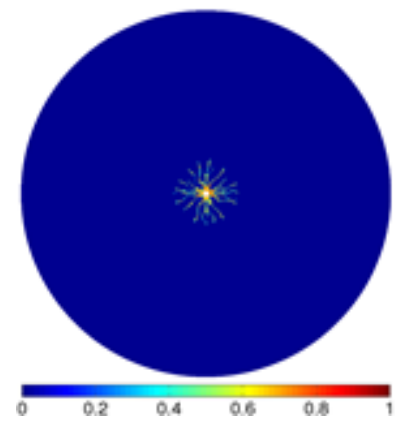

(a)

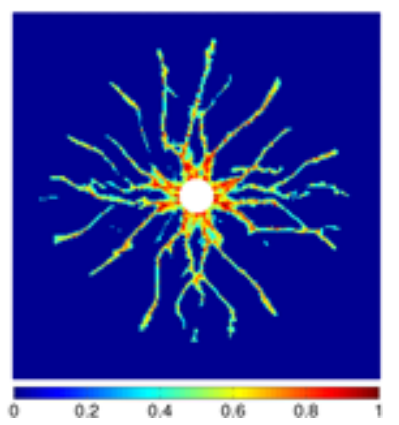

(b)

Figure 7: Subfigure (a): Contour of damage. Subfigure (b): Contour of damage in a $6 \mathrm{~m}$ by $6 \mathrm{~m}$ portion of the domain centered at the borehole. 


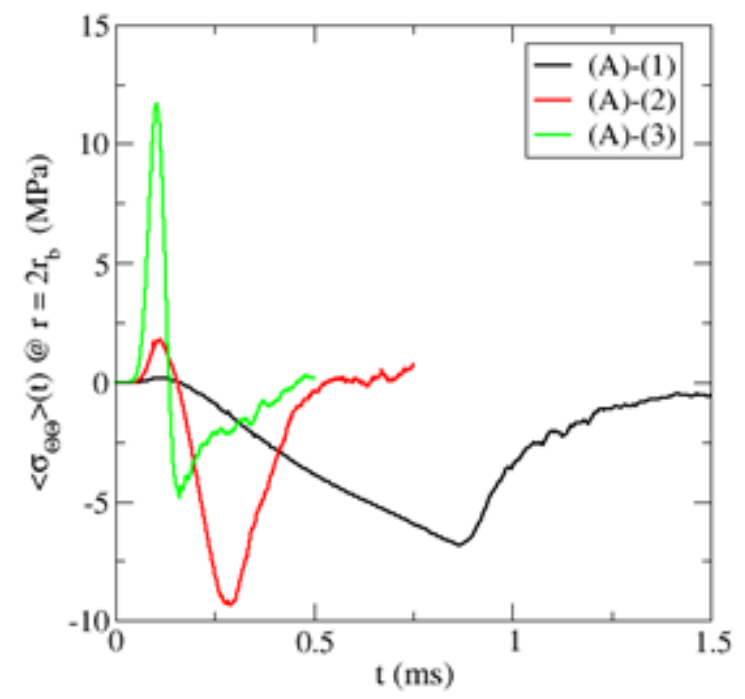

Figure 8: Time evolution of hoop stress averaged at $r=2 r_{b}$ for loading cases $(\mathrm{A})-(1,2,3)$.

\subsection{Influence of decay rate}

As discussed in the previous section, the number of fractures initiated from the borehole strongly depends on the initial loading rate. While the maximum fracture lengths are similar for cases $[(\mathrm{A})-(1,2)]$, the fractures obtained for case [(A)-(3)] are much shorter. Ultimate fracture extension is clearly sensitive to

50 the net impulse from loading, characterized by the area under the pressure-time curve shown in Fig. 3(b), The total impulse depends on the decay rate as well as the loading rate. Inspection of the temporal evolution of the damage for the three cases reveals that for the loading cases [(A)- $(1,2)]$, fractures initiate during the rise portion of the pressure pulse, whereas for case [(A)-(3)], fractures initiate

during the decay portion. For cases [(A)-(1,2)], the sustained borehole loading provides ample energy to the propagating fractures to continue propagation. For case $[(\mathrm{A})-(3)]$, the fractures initiate while the energy supply to the fracture tips is diminishing, resulting in earlier arrest and shorter fractures.

Shown in Fig. 10 are the pressure profiles and the corresponding contour plots for damage for cases [(A)- $(2,3,4,5)]$. As shown in the plots, the rise portion 


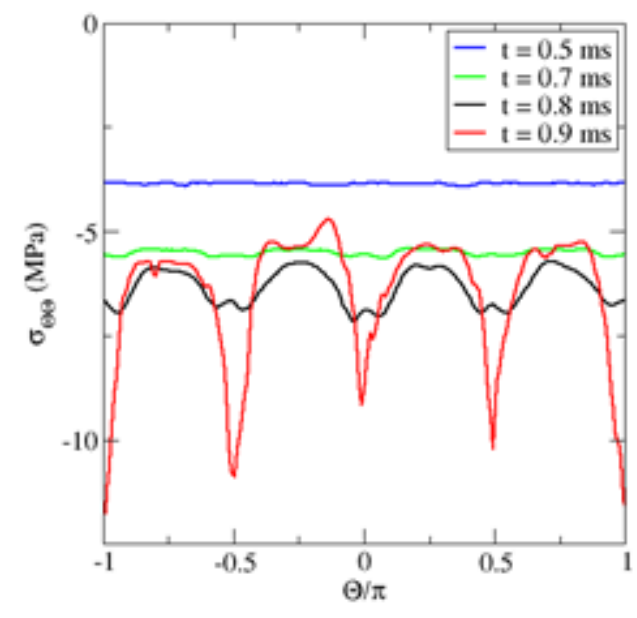

(a)

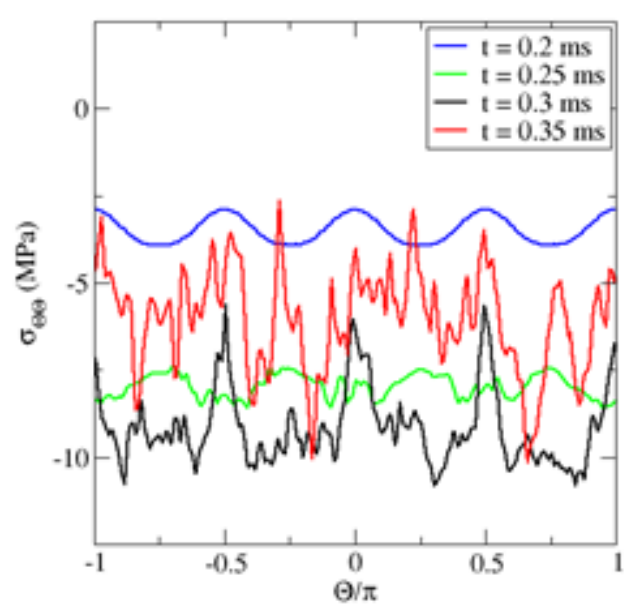

(b)

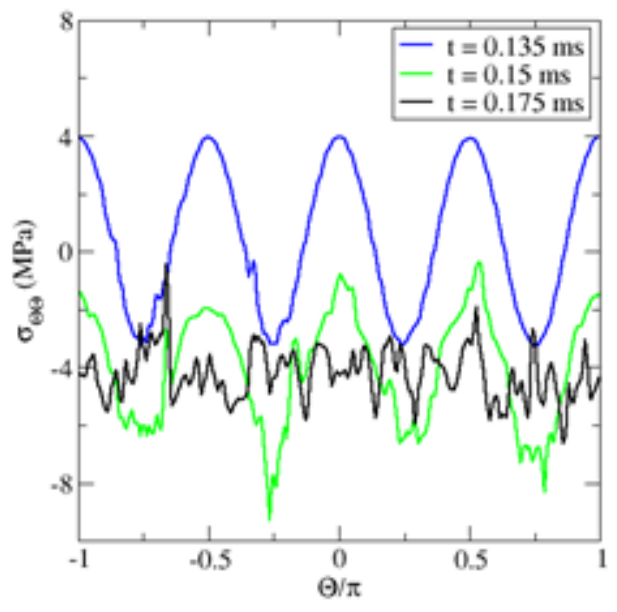

(c)

Figure 9: Time evolution of hoop stress at $r=2 r_{b}$ for loading case (a) (A)-(1), (b) (A)-(2), and (c) (A)-(3). In each case, time slices are chosen just prior to and after fracture initiation.

of the cases $[(\mathrm{A})-(4,5)]$ is similar to cases $[(\mathrm{A})-(2,3)]$, respectively. The decay rates for cases $[(\mathrm{A})-(4,5)]$ have been modified such that total input impulse for these cases is identical to that of case [(A)-(1)]. Comparison of the final fracture patterns obtained for cases $[(\mathrm{A})-(2)]$ and $[(\mathrm{A})-(4)]$ indicate that the ultimate 

obtained in case [(A)-(5)] are significantly longer than the corresponding case [(A)-(3)]. It is important to note that the initiation process in these cases are essentially identical. In case [(A)-(5)], the pressure on the borehole surface is held nearly constant after initiation. Clearly, the additional impulse imparted to highlight that while the number of fractures initiated primarily depends on loading rate, the ultimate fracture length depends on the net impulse imparted after initiation.

\subsection{Influence of in-situ stress state}

We now consider the influence of the in-situ stress state on fracture patterns. Comparisons are made between the initially unstressed cases [(A)-(1,2,3)] and those with isotropically compression of $10 \mathrm{MPa}$, cases [(B)-(1,2,3)]. Fig. 11 depicts the contours of damage for the cases $[(\mathrm{A}, \mathrm{B})-(1,2,3)]$ in a $10 \mathrm{~m}$ by $10 \mathrm{~m}$ portion of the domain centered at the borehole. A radial fracture pattern is obtained for both the initially unstressed and stressed cases for all loading rates. However, the fracture lengths are noticeably larger in the unloaded cases. This is expected because, if the system is initially under compression, a larger amount of energy is required to induce the system to a tensile state to initiate and propagate fractures. This effect is most evident for cases $[(\mathrm{B})-(1,2)]$ where the fracture lengths are only approximately $20 \%$ of the corresponding cases [(A)-(1,2)]. For case $[(\mathrm{B})-(3)]$ maximum fracture length is about $50 \%$ of case $[(\mathrm{A})-(3)]$. To further investigate the influence of in-situ stress on maximum fracture lengths, an additional simulation was performed using the same loading rate as case [(B)(2)], but using a different peak pressure of $300 \mathrm{MPa}$ and a rise time of $3 \mathrm{~ms}$. The fracture pattern for case [(B)-(2)]. The figures indicate that when the rock is under compression, for a given loading rate, longer fractures can be obtained by increasing the net impulse imparted to the system. The predictions also show that, for a constant net impulse, the in-situ stress limits the number of radial 

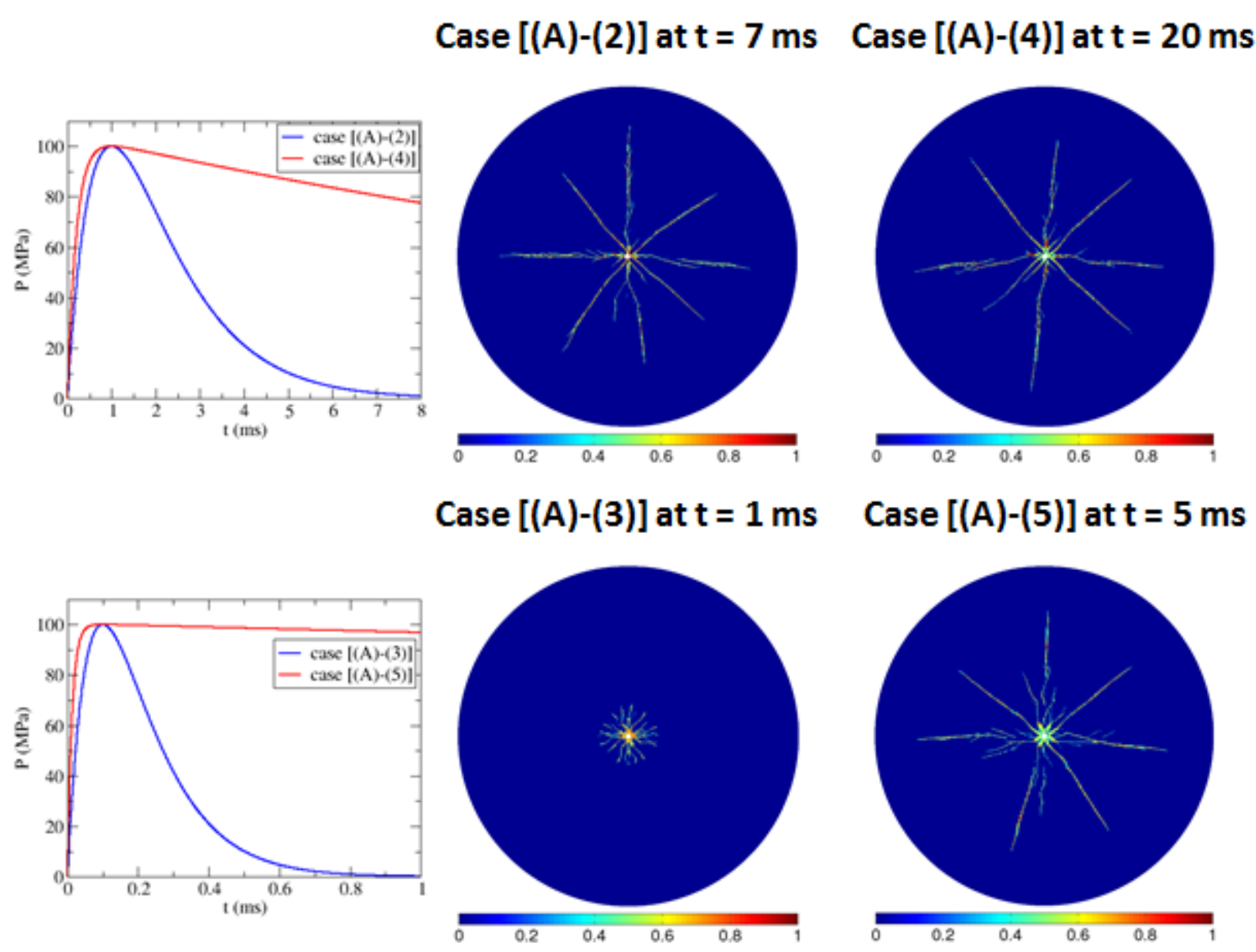

(a)

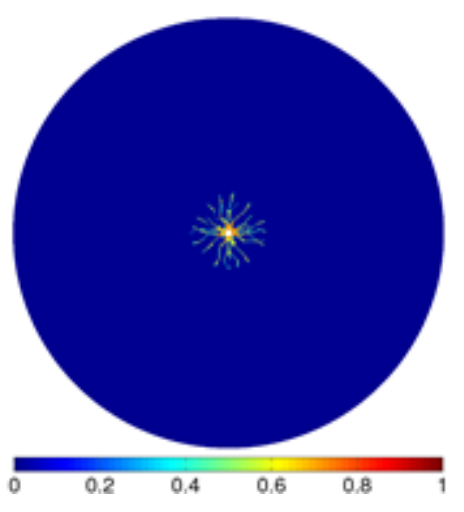

(b)

(c)

Figure 10: Effect of decay rate on fracture patterns. Subfigure (a): Time varying loading profile. Subfigure (b \& c): Contours of damage.

fractures initiated from the borehole.

The influence of the in-situ stress anisotropy on fracture patterns is demonstrated in Fig. 13. For all loading rates, the in-situ stress anisotropy has a consistent influence in the fracture paths. For an isotropic initial stress state, fractures initiate and propagate in all directions, as shown above. However, as stress anisotropy is increased, fracture extension in the $S H_{\max }$ direction is enhanced relative to the $S H_{\min }$ direction. For the fastest loading cases [(B,C,D)- 
(3)], the high rate of loading still induces multiple nucleation events for fracture initiation, resulting in comparatively more isotropic fracture patterns. In this sense, the high loading rate is able to partially overcome the orientational constraints imposed by the anisotropic confinement stresses. These plots illustrate that, if a system is under a large initial stress anisotropy, then initiating and propagating fractures using low loading rates will result in fractures primarily oriented perpendicular to the minimum confining stress direction, a similar situation to that expected in hydraulic fracturing. However, higher loading rates may be more successful in initiating and propagating fractures in multiple directions irrespective of the stress anisotropy. Nonetheless, fractures in the $\mathrm{SH}_{\min }$ direction may be expected to be shorter than $S H_{\max }$ direction.

\subsection{Comparison with other models}

We compare our numerical results with other similar experimental and numerical investigations, and emphasize that the following comparisons are only qualitative. More quantitative assessments of peridynamic models predictions in the context of dynamic fracture can be found elsewhere [19, 20]. Here, we consider results presented by Ma and An [31] and by Banadaki and Mohanty [32].

Ma and An utilize the Johnson-Holmquist (J-H) material model through LSDYNA to simulate blasting-induced rock fracture, and investigate the effects of loading rates on the fracture network generated using the function of Eq. (8). The J-H model [33] describes the strength of intact and fully fractured materials through an equation of state, and a damage evolution description . In their numerical simulations, they consider a square slice of rock six meters on a side

425 with a borehole 0.05 meters in diameter. Fig. (3c) from 31 is presented in Fig. 14(a).

Banadaki and Mohanty present both physical and numerical experiments of borehole fracture. They study explosive driven fracture of cylindrical rock samples of Barre granite, 0.144 meters in diameter and 0.150 meters in length with a borehole 6.45 millimeters in diameter. To separate out the effects of fracture induced by the radial blast wave from the fractures induced by the force 


\section{Case $[(A)-(1)]$ at $t=10 \mathrm{~ms}$ Case $[(B)-(1)]$ at $t=10 \mathrm{~ms}$}
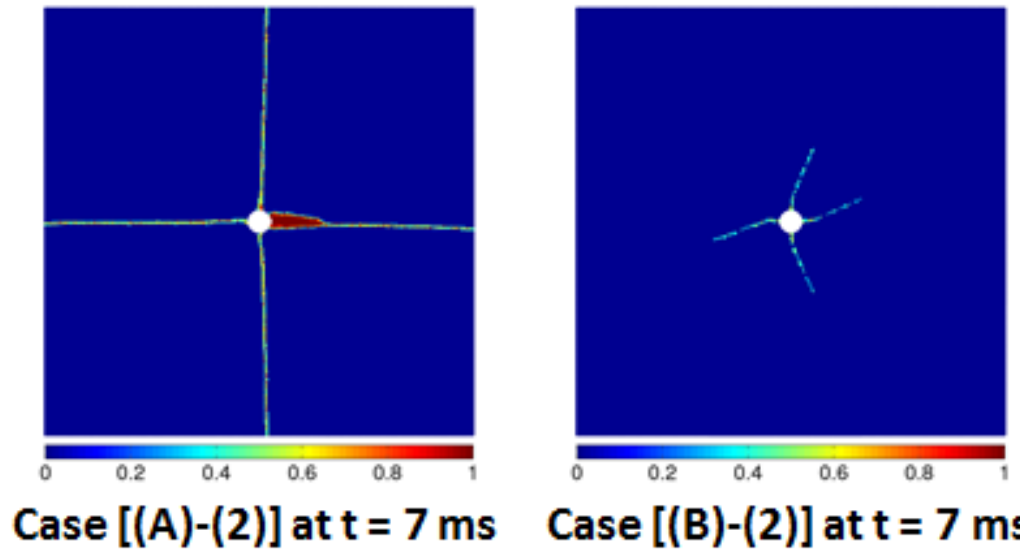

Case $[(B)-(2)]$ at $\mathrm{t}=7 \mathrm{~ms}$
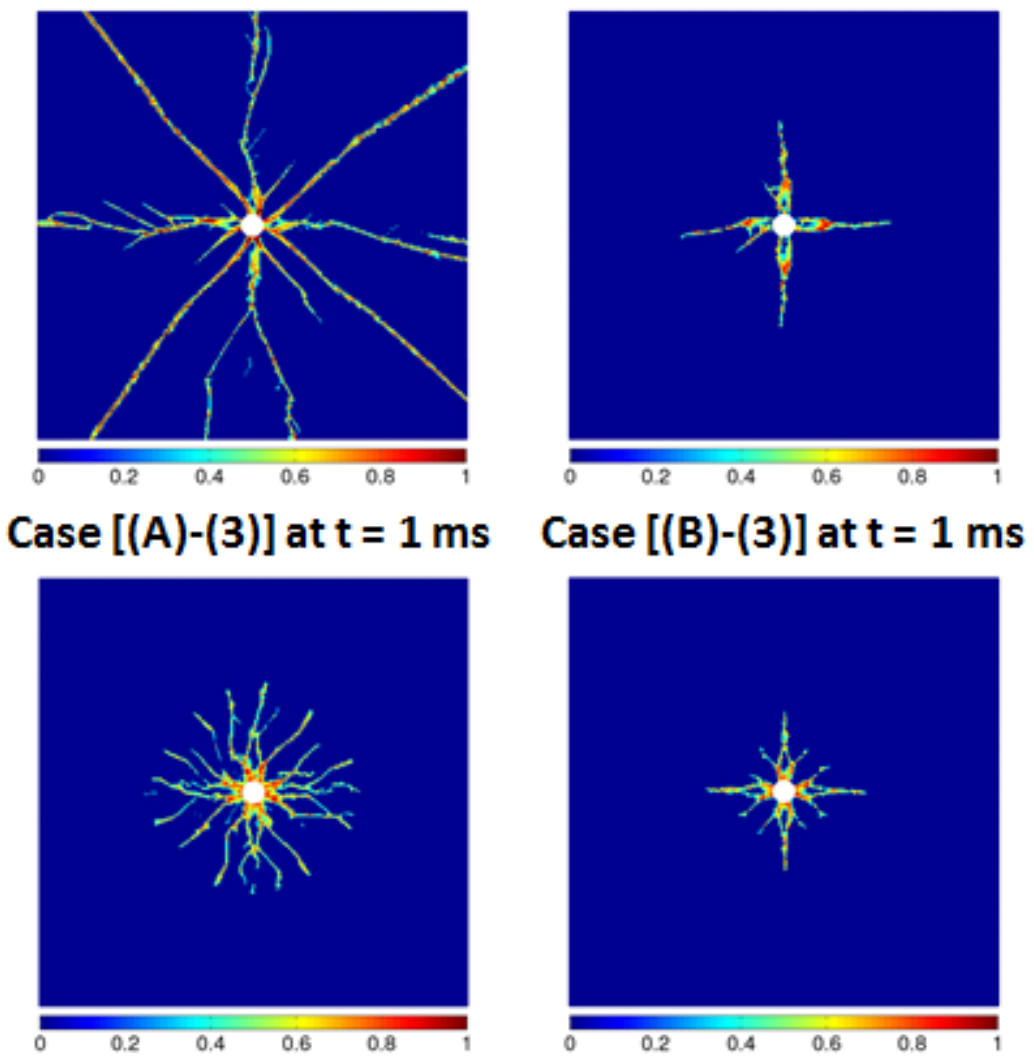

Figure 11: Contours of damage in a $10 \mathrm{~m}$ by $10 \mathrm{~m}$ portion of the domain centered at the borehole for cases [(A,B)-(1,2,3)]. 


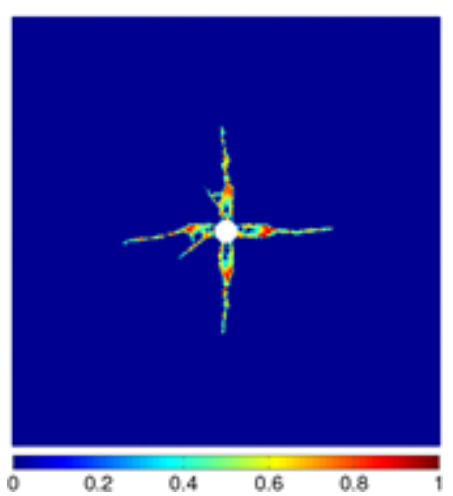

(a)

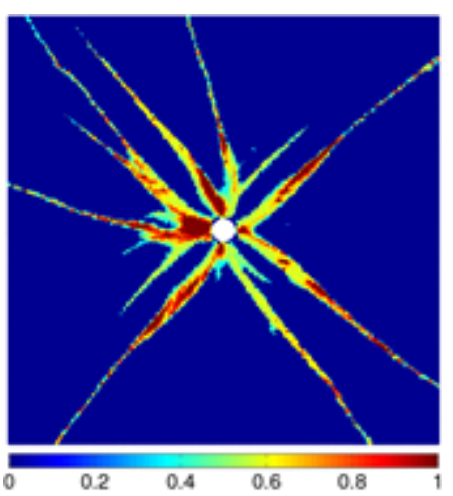

(b)

Figure 12: Damage contours in $10 \mathrm{~m}$ by $10 \mathrm{~m}$ domain around borehole with isotropic confining stresses. Subfigure (a) case [(B)-(2)] at $t=7 \mathrm{~ms}$, Subfigure (b) case [(B)-(2)] with $P_{0}=$ $300 \mathrm{MPa}$ and $t_{0}=3 \mathrm{~ms}$ at $t=10 \mathrm{~ms}$.

of explosion gasses flowing into the newly formed fractures, a copper tube was inserted into the borehole allowing transmission of the blast wave, but containing all gasses generated by the explosion. We note that the these experimental conditions are similar to the loading conditions in our studies. In their numerical simulations, they also use the $\mathrm{J}-\mathrm{H}$ model implemented within LS-DYNA. Figures (9a) and (9b) from 32 are shown in Figures 14(b) and 14(d), respectively. In the experimental image, (Fig. 14(d)) spall fractures are observed due to wave reflections off the free surface of the cylindrical sample. Characteristic peridynamic simulation results from the current study are shown in Fig. 14(c).

Comparing the results from the current study to those of Ma and An and Banadaki and Mohanty, we can make the following general observations.

Effects of loading rate upon the borehole fracture network: Ma and An report that for high loading rates $(20 \mathrm{MPa} / \mu \mathrm{s})$ only a crushed zone around the borehole is present. Moderate loading rates $(10 \mathrm{MPa} / \mu \mathrm{s})$ produce damage around the borehole accompanied by radial fractures. Low loading rates $(1 \mathrm{MPa} / \mu \mathrm{s})$ are reported to produce radial fractures. Analogous conclusions are observed under the current study, as reported in Fig. 7 
Case $[(B)-(1)]$ at $\mathrm{t}=10 \mathrm{~ms}$ Case $[(C)-(1)]$ at $\mathrm{t}=10 \mathrm{~ms}$ Case $[(D)-(1)]$ at $\mathrm{t}=10 \mathrm{~ms}$
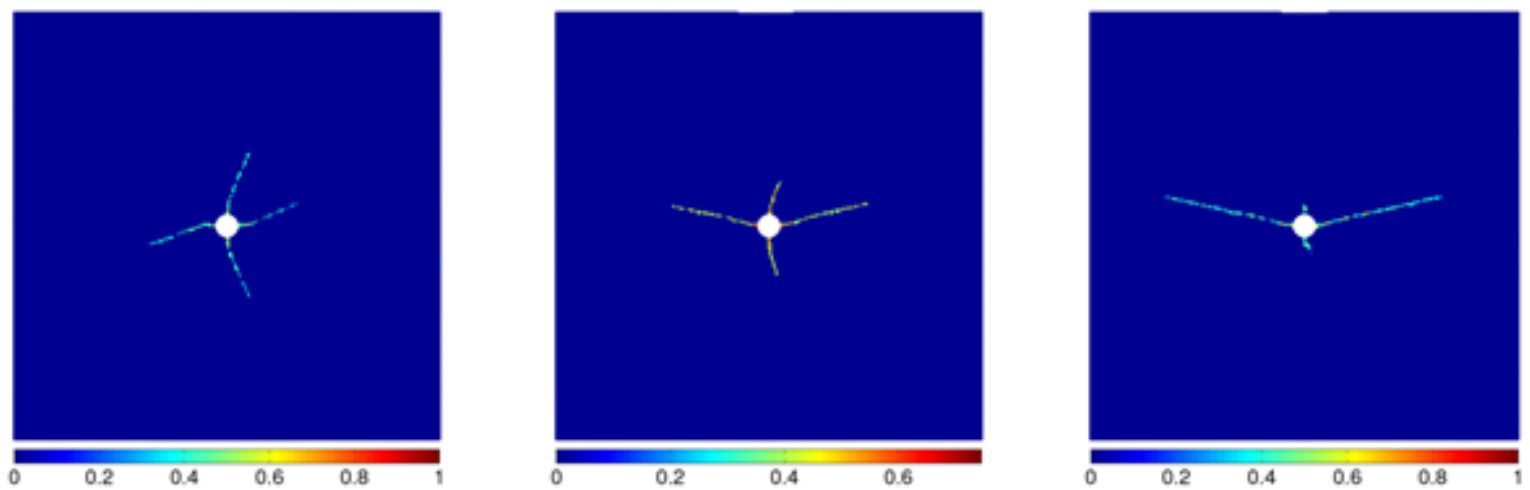

Case $[(B)-(2)]$ at $\mathrm{t}=7 \mathrm{~ms}$
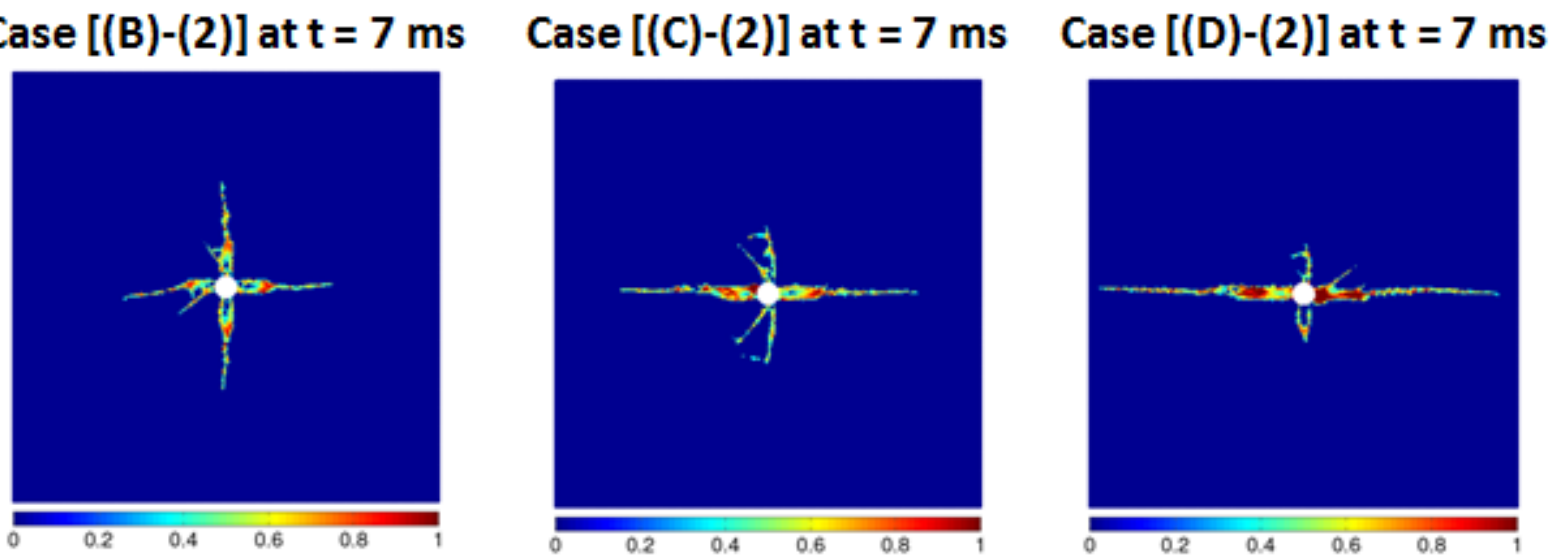

\section{Case $[(B)-(3)]$ at $\mathrm{t}=1 \mathrm{~ms}$}
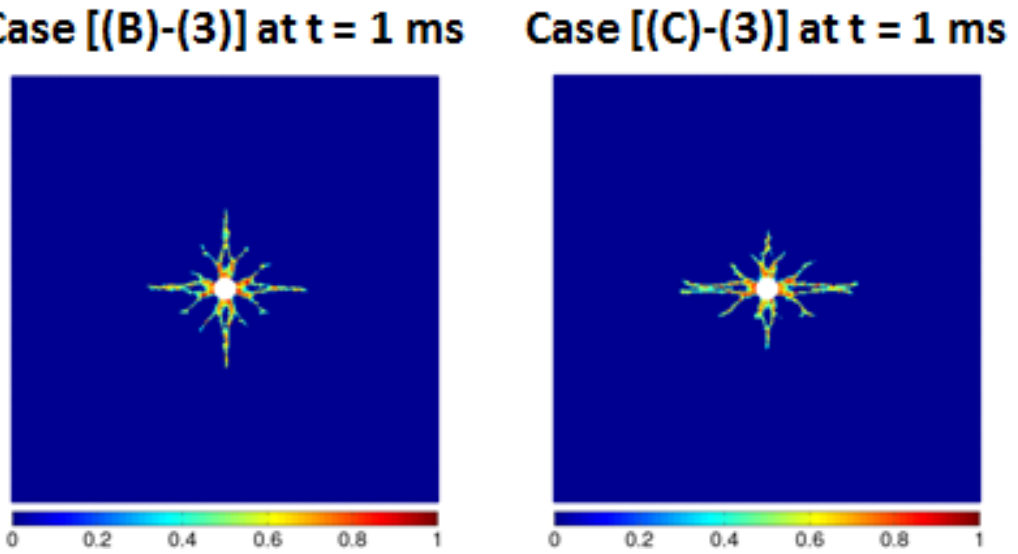

Case $[(D)-(3)]$ at $t=1 \mathrm{~ms}$
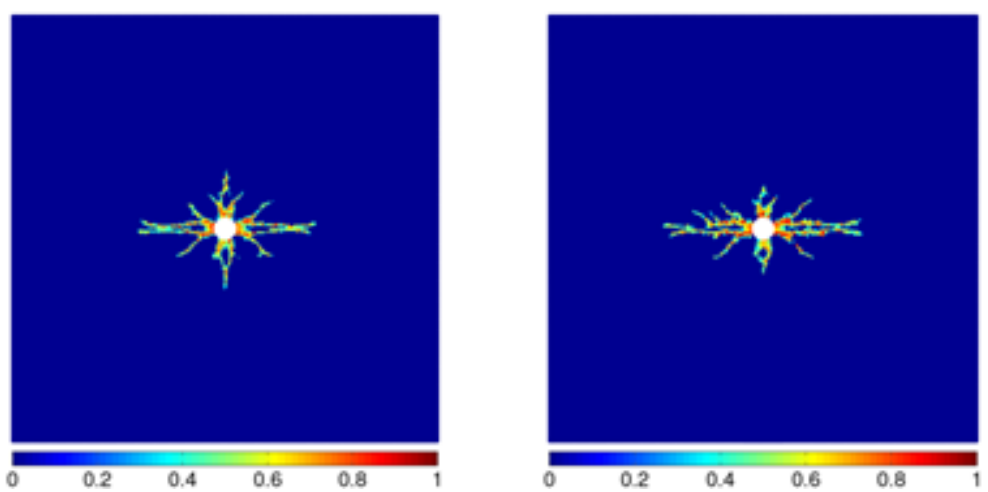

Figure 13: Contours of damage in a $10 \mathrm{~m}$ by $10 \mathrm{~m}$ portion of the domain centered at the borehole for cases $[(\mathrm{B}, \mathrm{C}, \mathrm{D})-(1,2,3)]$. 
This is conclusion is also supported by [7].

450

\section{Conclusions}

A novel non-local numerical called peridynamics was used to study the influence of loading conditions and in-situ stress on fracture initiation and propaga- 


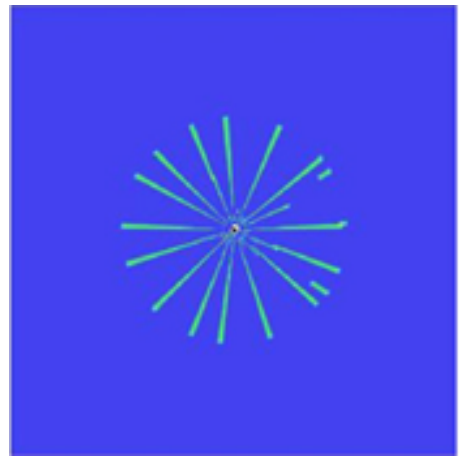

(a)

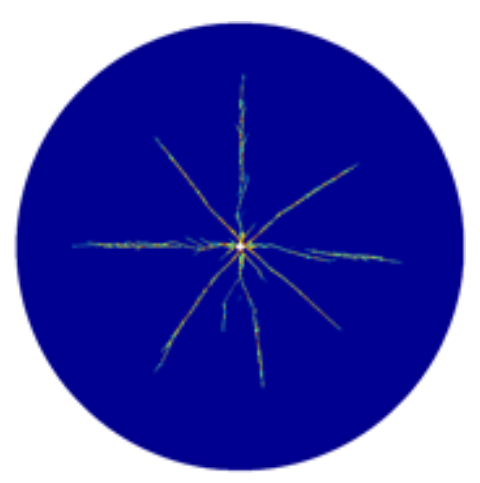

(c)

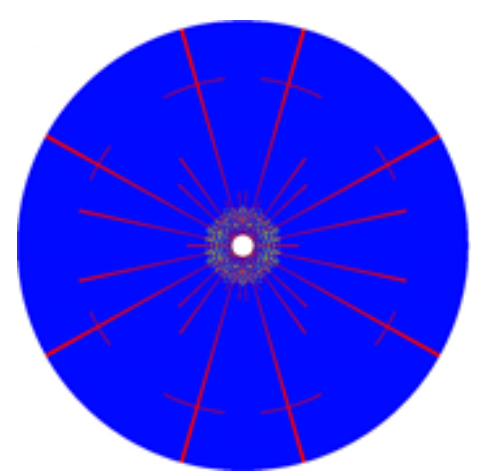

(b)

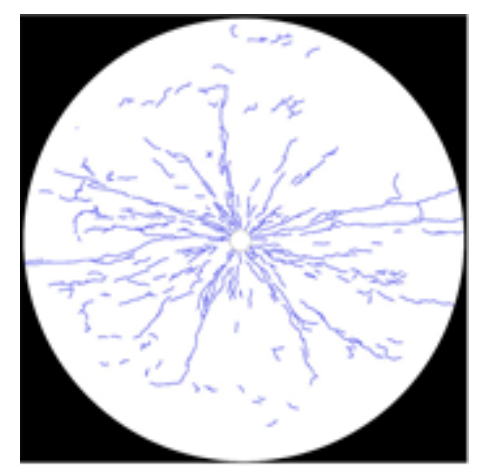

(d)

Figure 14: Characteristic simulation results from 31 (a) and 32 (b) using the J-H model in LS-DYNA show characteristic radial fracture patterns where the fractures do not turn or branch. Characteristic simulation results (c) from the current study show a fracture network with turning and branching fractures, in qualitative agreement with the experimental results (d) of 32. The authors would like to acknowledge Elsevier for allowing us to reprint the figures from 31 and 32 .

tion resulting from the dynamic loading of the surface of a borehole. Simulations were performed on a simplified rock geometry and the results were compared to relevant experimental and numerical studies found in the literature [12, 34].

The numerical results showed that the fracture patterns were significantly influenced by the loading rate as well as the total impulse applied. A radial fracture pattern was obtain in all the cases; fractures initiated close to the bore- 
hole surface and propagated radially outwards. Slower loading rates produced fewer and longer radial fractures, while higher loading rates produced more radial fractures accompanied by fracture branching and a uniform zone of large damage near the borehole surface. The size of damage zone increased with increasing loading rate.

For all loading conditions fractures arrested during the decay portion of the pressure pulse. Predictions showed that the final fracture length depended both loading rate and the net impulse applied to the borehole. Fractures that initiated during the rise portion of the pressure pulse were longer than those that initiated during the decay portion. Furthermore, predictions also showed that, for some conditions, sustained pressure on the borehole surface after the rise time produced significantly longer radial cracks than when pressure decayed 495 rapidly.

Our simulations indicate a strong effect of confining stresses. Application of in-situ stress decreased the number of radial fractures, attenuated their propagation, and limited fracture branching. Numerical results showed that the fracture extensions are largest in the direction perpendicular to the minimum compressive stress for the range of loading rates used in this study.

Our simulations highlight the controlling factors on fracture initiation, branching, and extension that results from the interplay of loading rate, confining stress, wave propagation, and energy dissipation in brittle materials. It opens doors for optimizing loading conditions to maximize the creation fracture net505 works for well-stimulation applications, including the possibility of employing multiple pressure pulses for fracture creation, or utilizing a pressure pulse that combines an initially high loading rate followed by a much slower loading to enhance fracture extension. In the latter case, it may be expected that a high loading rate would initiate multiple fractures and condition the near borehole rock, such that subsequent application of a lower loading rate pulse on the fracture faces would further extend these fractures to obtain a highly branched isotropic dense fracture network. Peridynamics provides a very convenient and robust framework to explore these ideas. 


\section{Acknowledgment}

515 supporting this research and permitting us to publish this work. Sandia is a multiprogram laboratory operated by Sandia Corporation, a Lockheed Martin Company, for the U.S. Department of Energy under contract DE-AC04-94AL85000.

[3] RP Swift, AS Kusurov. Multiple fracturing of boreholes using tailored-pulse loading. Soc Petrol Eng J. 1982; 22: 923-932.

[4] WL Fourney, DB Barker, DC Holloway. Model Studies of Well Stimulation Using Propellant Charges. Int J Rock Mech Min Sci. 1983; 20: 91-101.

[5] DD Keogh, DR Curran. Applicability of Tailored Pulse Loading for Gas Well 530

[6] WC Hunt, WR Shu. Controlled Pulse Fracturing for Well Simulation. Society of Petroleum Engineers. 1989;

[7] FV Donzé, J Bouchez, SA Magnier. Modeling fractures in rock blasting. Int J Rock Mech Min Sci. 2004; 34: 1153-1163.

[8] HK Kutter, C Fairhurst. On the fracture process in blasting. Int J Rock Mech Min Sci. 1971; 8: 181-202.

[9] S McHugh, D Keough. Use of laboratory-derived data to predict fracture and permeability enhancement in explosive-pulse tailored field tests. Issues in 
Rock Mechanics: Proceedings of the symposium on Rock Mechanics. 1982; 23: $504-514$.

[10] JF Cuderman. Multiple fracturing experiments-propellant and borehole considerations. Society of Petroleum Engineers. 1982;

[11] NR Warpinski, RA Sehmidt, PW Cooper. High energy gas fracturing: multiple fracturing in a wellbore. Proceedings of 20th US symposium on rock mechanics. 1979; 143-152.

[12] SH Cho, K Kaneko. Influence of the Applied Pressure Waveform on the Dynamic Fracture Processes in Rock. Int J Rock Mech Min Sci. 2004; 41: $771-784$.

[13] SG Chen, J Zhao. A study of UEDC modeling for blasting wave propagation in joint rock mass. Int J Rock Mech Min Sci. 1998; 35: 93-99.

[14] A Fakhimi, M Lanari. DEM-SPH simulation of rock blasting. Comput Geotech. 2014; 55: 158-164.

[15] GW Ma, H Hao, YX Zhou. Modeling of wave propagation induced by underground explosion. Comput Geotech. 1998; 22: 283-303.

[16] CA Duarte, ON Hazmeh, TJ Liszka, WW Tworzydlo. A Generalized Finite Element Method for Simulation of Three-Dimensional Dynamic Crack Propagation. Comput Method Appl M. 2001; 190: 2227-2262.

[17] SA Silling. Reformulation of elasticity theory for discontinuities and longrange forces. J Mech Phys Solids. 2000; 48: 175-209.

[18] SA Silling, E Askari. A meshfree method based on the peridynamic model of solid mechanics. Comput Struct. 2005; 83: 1526-1535.

[19] YD Ha, F Bobaru. Studies of dynamic crack propagation and crack branching with peridynamics. Int J Frac. 2010; 162: 229-244. 
[20] YD Ha, F Bobaru. Characteristics of dynamic brittle fracture captured with peridynamics. Eng Fract Mech. 2011; 78: 1156-1168.

[21] SA Silling, M Epton, O Weckner, J Xu. Peridynamic States and Constitutive Modeling. J Elasticity. 2007; 88: 151-184.

[22] JT Foster, SA Silling, WW Chen. Viscoplasticity using peridynamics. Int J Numer Meth Eng., 2009; 81: 1242-1258.

[23] O Weckner, N Abdullah, N Mohamed. Viscoelastic material models in peridynamics. Appl Math Comput. 2013; 219: 6039-6043.

[24] SA Silling, RB Lehoucq. Peridynamic Theory of Solid Mechanics. Adv Appl Mech. 2010; 44: 73-168.

[25] SA Silling, RB Lehoucq. Convergence of Peridynamics to Classical Elasticity Theory. J Elasticity. 2008; 93: 13-37.

[26] R Panchadhara, P Gordon. Application of peridynamic stress intensity factors to dynamic fracture initiation and propagation. Int J Frac. 2016; 201: $81-96$.

[27] JC Jaeger, NGW Cook, RW Zimmerman. Fundamentals of Rock Mechanics. 4th ed. Blackwell Publishing; 2007.

[28] ML Parks, RB Lehoucq, SJ Plimpton, SA Silling. Implementing Peridynamics within a Molecular Dynamics Code. Comput Phys Comm. 2008; 179: $777-783$.

[29] S Plimpton. Fast Parallel Algorithms for Short-Range Molecular Dynamics. J Comput Phys. 1995; 117: 1-19.

[30] RB Lehoucq. Force flux and the peridynamic stress tensor. J Mech Phys Solids. 2008; 56: 1566-1577.

[31] GW Ma, X An. Numerical simulation of blasting-induced rock fractures. Int J Rock Mech Min Sci. 2008; 45: 966-975. 
[32] MMD Banadaki, B Mohanty. Numerical simulation of stress wave induced fractures in rock. Int J Impact Eng. 2012; 40: 16-25.

[33] GR Johnson, TJ Holmquist. An improved computational constitutive model for brittle materials. AIP Conference Proceedings. 1994; 309: 981-984.

[34] Z Zhu, B Mohanty, H Xie. Numerical Investigation of Blasting-Induced ${ }_{595} \quad$ Crack Initiation and Propagation in Rocks. Int J Rock Mech Min Sci. 2007; 44: $412-424$. 